\title{
Neuroradiology, Anesthesia, Bioengineering, and Hardware Programming in the Clinical Applications of Deep Brain Stimulation
}

\author{
Ayden Jacob ${ }^{1}$, Krishnan Chakravarthy ${ }^{2}$ \\ 1. Interventional Radiology and Oncology, UCSF School of Medicine 2. Department of Anesthesia, \\ Critical Care and Pain Medicine, University of California, San Diego
}

$\square$ Corresponding author: Ayden Jacob, brainanalytics1@gmail.com

Disclosures can be found in Additional Information at the end of the article

\section{Abstract}

Deep brain stimulation (DBS) is an interventional surgical procedure used to treat an array of sensorimotor, cognitive, and psychiatric diseases originating in distinct brain regions. Although the Food and Drug Adminstration has approved DBS for the treatment of essential tremor and Parkinson disease, this technique is currently being used to treat major depression, dystonia, and obsessive compulsive disorder. Pathophysiological brain areas responsible for abnormal functions are targeted with implanted electrodes that are subsequently attached to battery packs to provide targeted electrical stimulation within the necessary parameters of stimulation required to decrease the phenotypic expressions of these debilitating diseases. DBS regularly targets the stimulation of the ventral thalamic nuclei, subthalamic nucleus, globus pallidus and the basal ganglion in an attempt to diminish symptoms of tremor, Parkinson disease, Tourette syndrome, and pain. Herein, we aim to address the bioengineering that dictates the limitations of current DBS treatments, as well as the history, current use, and potential of DBS as it relates to a variety of motor and neuropsychiatric diseases. This will serve as an insightful analysis about the current landscape of DBS and its potential for more widespread applications in the clinical arena.

Received 03/21/2014 Review began 03/29/2014 Review ended 04/17/2014 Published 04/21/2014

C) Copyright 2014

Jacob et al. This is an open access article distributed under the terms of the Creative Commons Attribution License CC-BY 3.0., which permits unrestricted use, distribution, and reproduction in any medium, provided the original author and source are credited.
Categories: Psychiatry, Radiology, Neurosurgery

Keywords: deep brain stimulation, biomedical engineering, intracranial, Neurosurgery, functional neurosurgery, neuroscience, electrosurgery

\section{Introduction And Background}

\section{Neurophysiology of deep brain stimulation}

Deep brain stimulation offers a method by which a surgeon may affect neuronal discharge patterns within the vicinity of the target, as well as in distant regional sections of the brain, via conveying bioelectric impulses along trajectories that originate in the target area and spread to remote neuronal structures [1]. In DBS, stereotactically-guided electrodes are placed within a predefined target structure in the brain, and electrical stimulation of these electrodes pulses specific neurons causing a bioelectrical effect in the targeted brain cells. Although the precise mechanism of action of DBS remains elusive, it is clinically accepted that neuronal signaling and bio-molecular function is influenced by electrical stimulation of the brain. Great controversy exists as to the effects of DBS on brain function, as well as its safety and efficacy in the treatment of different neurologically-based disorders. Adding to the uncertainty is the fact 
that the neuroscientific community has clearly demonstrated that different neurons demonstrate a variety of ion channels that possess a variety of voltage-sensitive activation and inactivation parameters. Kern, et al. report that the effects of DBS are frequency-dependent and are best realized at $>100 \mathrm{~Hz}$, while no therapeutic relief is seen at $<50 \mathrm{~Hz}$ [2]. The relationship between frequency output and neuronal response was originally demonstrated in the 1960s by Hassler, et al. [3]. Further, the stimulation pulse emanating from the electrodes determines the specific neuronal elements that will be altered by this impulse; the cell soma is predominantly influenced by longer pulse widths, while shorter pulses are known to impact the molecular and biochemical characteristics of axons. Consequently, the manner by which DBS influences the functionality of individual neurons and neuronal circuits remains elusive. However, the FDA approved DBS in 1997 for the treatment of tremor and PD-related tremor. In 2003, the FDA approved DBS for subthalamic nucleus stimulation and globus pallidus internus stimulation in the treatment of Parkinson's disease, followed by an approval in 2009 to treat obsessivecompulsive disorder (OCD). The emerging method by which DBS may be used to treat a host of brain-based diseased is an exciting field which unites basic science, engineering and clinical medicine.

With the advent of human stereotaxic devices in the late 1940s, neurosurgeons were enabled to utilize electrodes within the brain to investigate the primary effects of deep brain stimulation on deep structures within the white matter [4]. In the 1970s, several reports began demonstrating clinical attempts at utilizing implanted electrodes to treat chronic pain [5], movement disorders, and epilepsy [6]. Through the 1980s, further studies began introducing the use of DBS for the treatment of a variety of movement disorders [7]. The introduction of computational biotechnology in the last three decades has enabled the field of deep brain stimulation to grow with unprecedented success. Novel introductions into the fields of neuropsychiatry, cognitive behavior disorders, movement disorders, and neuroelectrical-based diseases have all been influenced and treated by the innovations brought about through DBS. Importantly, Perlmutter, et al. outlines various elements that are involved in the efficacy and potency of electrical input into narrowed areas of the brain. These include the amplitude of the stimulation, physiologic properties of the neurons being stimulated, geometry of the stimulus field, the geometry of the targeted elements, and even the underling pathophysiology of the particular disease state tissue being treated [8]. Herein, we aim to outline the neurophysiological circuits that are positively targeted for the treatment of a host of diseases via deep brain stimulation, and outline the engineering, software, and hardware mechanisms as well as imaging technologies utilized to implement this advanced technology in brain treatment delivery.

\section{Review}

\section{Deep-brain stimulation of the basal ganglia}

The basal ganglia is intrinsically involved in voluntary motor control, procedural learning, reward sensation and prediction, motivation and goal formation, sensorimotor associative learning, decision-making, and executive function [9-10]. The malfunction of the basal ganglia and its connective circuits is a root cause for a variety of neurological disorders [11]. The structures of the basal ganglia are ideal sites of target for therapeutic interventions for disorders ranging in motor abnormalities and cognitive functions [12]. Early stage treatment of disorders affecting the basal ganglia tend to rely heavily on medications to alter synaptic transmission within its circuits. Although pharmacologic interventions aimed at curing disorders emanating from irregular activity of the basal ganglia are somewhat successful, the side-effects they inflict on the patient are intolerable. Further, due to the habituated nature of neurons, an everlasting increase of pharmacologic dosage is required to obtain the same effect as the disease progresses. To circumvent this issue, researchers and surgeons have developed a surgical procedure to therapeutically modulate basal ganglia circuits via electrical current 
injection [13]. This method, called deep-brain stimulation (DBS), has been adapted to treat medically-intractable cases of various neurological and neuropsychiatric disorders. In demonstrating alterations within the brain in diseases such as Parkinson's disease (PD), dystonia, major depression, Tourette syndrome (TS), and obsessive-compulsive disorder (OCD) [13-17], the involvement of the basal ganglia, in addition to other neuronal circuits, will be clarified.

\section{Parkinson's Disease}

PD is a debilitating disease in both a societal and physical sense. Phenotypic expression occurs in patients during late middle age; yet, there are some rare cases which begin in childhood. PD tends to give patients symptoms including slow motor responses, an inability to initiate voluntary movements, uncontrollable tremor, and in more progressed cases, poor short-term memory, a diminished learning ability, depression-like symptoms, and even psychosis [12-13, 18]. PD is characterized by the progressive degeneration of midbrain dopaminergic neurons of the substantia nigra pars compacta (SNc). Consequently, a significant loss of dopaminergic innervation to the dorsal striatum in a posterior putamen to anterior caudate progression is evident in the brains of PD patients [19]. This degeneration causes many pathological effects to cortico-striato-thalamo-cortical (CSTC) circuits. Most notably, such circuits exhibit poor correlation in neuronal activity within the dorsal striatum, globus pallidus interna (GPi), thalamus, and motor cortex, as would be expected in CSTC circuits with poor signal-to-noise contrast. Additionally, the frequency of oscillatory activity in these neuronal circuits are impeded in PD, resulting in poor initiation of voluntary motor behaviors. It is theorized that the phenotypic motor symptoms in PD are due to the inability of a disruptive basal ganglia network to amplify and specify directed motions and behavior [15, 19].

Deep brain stimulation (DBS) is associated with significant improvement of motor complications in patients with severe Parkinson's disease after some six to 12 months of treatment [20]. Depending on the site of stimulation, the clinical effects of DBS in PD differ [8]. Limb tremor may be significantly reduced by thalamic stimulation in the region of the ventral intermediate nucleus (VIM) [21]. Research has shown that stimulation of the GPi may reduce all of the motor manifestations of PD, including 'the reduction of dopa-induced dyskinesias' [22]. According to studies done by Loher, et al, DBS stimulation of the GPi may also be useful in treating painful cramps and sensory symptoms associated with doses of levodopa [23]. A limitation of GPi stimulation is that it does not permit the reduction of pharmacological reduction in PD, and thus may not be as useful to patients interested in reducing their medication intake due to unwanted side-effects. However, patients receiving GPi DBS have also shown marked improvements in cognitive symptoms [13].

Subthalamic nuclei (STN) stimulation provides a reduction in motor symptoms in PD patients [24]. Several studies have demonstrated that bilateral STN DBS improves tremor, physical posture, and bradykinesia [25-27]. PD patients receiving DBS to either the STN or GPi have shown significant alleviations of movement latency, inhibition of voluntary movements, tremor, and other motor-oriented symptoms [28]. A comparative study attempting to demonstrate phenotypic differences from STN DBS to that of GPi DBS failed to show significant clinical differences between the two groups, even in regards to changes in medication concentrations needed to treat PD [22]. Although DBS is currently used to alleviate the symptoms of PD, the positive effects are transient, as current stimulation does not prevent further degeneration of SNc neurons, which drives the progression of the disease to later stages. DBS therapy for PD is known to be efficacious for a 10 year maximum period, after which patients may see a decline in their health and recurrence of their symptoms [29]. Engineering new methodologies, which can be coupled with DBS treatment to both stimulate and sustain the abnormal neurons, will be an area of research that may potentially change the manner in which we treat patients with PD in the future. 
Although bioengineering methods have advanced the utilization of electrode implantation in PD patients' brains, the precise mechanism by which it relieves symptoms remains elusive. Nevertheless, the outlined components of DBS activity in brain matter are the most commonly accepted theories, through which DBS inhibits activation of cell bodies at both STN and GPi targets, as well as stimulates activation of efferent axons of both the STN and GPi. [13, 15].

In comparing the effects of STN and GPi stimulation with DBS, STN excitation is associated with impairment of particular cognitive processing skills. When utilizing stimulation to control motor irregularities, impairments of spatial delayed responses and delayed recall have been reported [30]. Further differences between stimulation of the GPi and STN illustrate a potential improvement in executive functions with STN stimulation [31]. Interestingly, STN DBS stimulation in PD has been associated with infrequent production of unacceptable emotional responses to a given stimulus, including manic responses [32], hallucinations [33], and decreased mood [34]. The advantageous effects of DBS in alleviating PD symptoms may be attributed to a variety of neuropathological alterations, which manifest from electrical stimulation of the brain. A proposed theory hypothesizes that the alleviation of symptoms results from a stronger activation of the indirect pathway in the dorsal basal ganglia circuit. Accordingly, CSTC circuits directing voluntary motor behaviors would have greater contrast to those that are not pertinent [35]. A theory which is gaining more acceptance within the biomedical community as to the effects of DBS in PD states that DBS overrides the pathological activity patterns of the basal ganglia, thereby reestablishing higher-frequency oscillatory activity within CSTC circuits. This specific hypothesis is strengthened by evidence illustrating that nigrostriatal, pallidothalamic, pallidonigral, and various other imperative neuronal fiber tracts are also activated by DBS [13]. Overall, although the precise mechanism by which DBS affects neuronal output in a positive matter, it is accepted that a synergistic effect of DBS within brain matter is responsible for symptomatic improvements in PD patients [15].

\section{Dystonia}

A clinical syndrome categorized by intermittent and sustained muscle contractions, dystonia generates involuntary muscular movements and abnormal skeletal postures. Dystonia can be caused by mutations in the DYT1 gene, yielding adverse effects of dopamine-blocking agents, or severe neural damage due to ischemia or traumatic brain injury [36-37]. EMG recordings of dystonic patients exhibit an involuntary overexcitation of inappropriate and opposing muscles. Such overexcitation is also seen in neural activity of the spinal cord, primary motor cortex, and thalamus, while there is a substantial decrease in tonic activity of the GPi [36]. This pathophysiology clearly elucidates dystonia as a disorder of the basal ganglia. With reduced GPi inhibition of the thalamus, the indirect pathway of the dorsal basal ganglia circuit is severely weakened, thereby disinhibiting circuits directing non-pertinent motor behaviors. As a result of poor signal-to-noise contrast in motor circuits, unintended muscle contraction can occur frequently [36]. This physically debilitating disease of the motor circuitry could be treated with botulinum toxin injections, but pharmacological intervention is of limited use due to the severity and consistency of the muscle contractions. The advent of stereotaxic surgery in the 1950s has been introduced as a sustainable method of treatment for dystonia [36]. Surgical approaches, such as thalamotomy [37] and pallidotomy [38], have been tested in the treatment of dystonia but have induced unwanted responses and severe side-effects influencing speech and cognition [39]. Thus, the introduction of deep brain stimulation targeting the internal globus pallidus is an attractive mode of therapy as it is a reversible procedure with low morbidity [40-44]. In studies conducted by Vitailt, et al., dystonia patients showed significant improvement post-deep brain stimulation of the globus pallidus. This study reported the improvement in mean dystonia movement scores of 51 percent, while one-third of the patients had significant improvements of more than 75 percent relative to preoperative functional scores. According to Vercuel, et al., different outcomes from DBS administration in dystonia patients may be allocated to the specific genetic abnormalities underlying the disease in each 
patient, as well as the specific location of the electrodes in the brain matter [45]. In a controlled trial conducted in 2005, DBS was administered to 22 patients with dystonia, and a significant 30\%-50\% improvement in symptoms was detected [46]. Kiss, et al. have also demonstrated initial promising results for the treatment of primary cervical dystonia, and Castelaneu, et al. reported success in the treatment of secondary dystonia [47-48].

\section{Major Depression}

The incidence of depression continues to increase, while psychopharmacologic intervention efficacy remains stagnant. Major depression is a leading cause of disability in the U.S., as it disrupts patients' lives by inducing decreased energy levels, abnormal mood swings, low energy, and a loss of hope and motivation [3, 17]. The underlying etiology of major depression remains elusive and highly debated. Presumed neuropathophysiology involved in this mental health disorder include a neurotransmitter concentration imbalance of serotonin, dopamine, and even norepinephrine. Further, anatomical regions specific to phenotypic expressions of major depression have been known to include the ventromedial prefrontal cortex (VMFPC), anterior cingulate cortex (ACC), and the subcallosal cingulate gyrus (SCG) [49]. Clearly, the subcortical interconnectivity between neuronal regions involved in major depression is vast, and thus marking a particular region for DBS intervention has evolved overtime. The SCG provides a known connectivity between the amygdala, hypothalamus, VMPFC, and ACC, and thus was targeted as one of the first DBS sites of treatment for major depression [50]. Patients refractory to conventional treatment methods for depression who have undergone SCG DBS have presented with increased mood, decreased anxiety, and significant increases in sleep cycle rhythms [50-51]. Functional analysis of the brain revealed that SCG DBS also causes downstream structures to emulate an activity pattern similar to that observed when antidepressant medications are working, although it is unknown whether the change in activity in these brain regions causes improvement in depressive symptoms or the change is merely a biomarker for improvement [50].

The nucleus accumbens (NAcc) has also been potential a target for DBS therapy in the treatment of mood disorders like major depression because of its involvement in the brain's reward and motivation circuit $[9,52]$. NAcc DBS has also been shown to markedly improve depressive symptoms in patients by causing marked functional changes in specific neuroanatomic regions implicated in the pathophysiology of the disease, such as the ACC, VMPFC, and thalamus [17, 52]. The precise molecular and electrical mechanism by which NAcc DBS improves symptoms in major depression is not fully understood, but given the role of NAcc in motivation and mood, it is hypothesized that stimulation of the NAcc assists in overriding neuronal abnormalities in these regions responsible for low motivation and depressed mood [53].

\section{Anesthetic considerations for performance of deep brain stimulation placement}

We present here a short synopsis of the key elements that are critical when performing deep brain stimulation under various forms of regional and general anesthesia. We refer the readers to the review by Poon, et al. for a more comprehensive discussion regarding anesthetic management of deep brain stimulation procedures [54]. The primary aim remains to provide optimal surgical conditions, conduct appropriate intraoperative monitoring using standard ASA monitors, including neuro-monitoring for target localization, and to diagnose and treat any intraoperative and postoperative complications.

Preoperative Setting 
In the preoperative setting, critical elements to consider in regards to developing a risk-tobenefit ratio of performing the surgery should include assessment of increased bleeding risk, i.e. underlying coagulopathy and uncontrolled hypertension. Specific emphasis should be placed on past anesthetic history and any associated cardiac and pulmonary issues that may predispose patients to a complicated response to general anesthesia. In addition, other intraoperative risks include exposure to MRI using a full body radiofrequency (RF) coil and patients who may require shortwave, microwave, or therapeutic ultrasound diathermy. Discussions with the primary surgical team regarding the benefits of the DBS procedure should include individual patient clinical demographics, with specific emphasis on whether the patient has underlying dementia, or will have an ability to operate the neurostimulator once it is successfully placed. In particular, since the occurrence of dementia is a common problem in patients with Parkinson's disease, our recommendations are in line with Baltuch, et al. [55] which state Mini Mental Status Exam (MMSE) of <24 or Mattis Dementia Rating Scale (MDRS) total score of $<120$ would suggest the patient is a poor surgical candidate. Other specific issues to be addressed are whether the patient will tolerate the 'awake' technique and will be able to cooperate with the surgery team and the anesthetist in the operating room during the 'awake' component of the procedure. Specific emphasis in regards to the preoperative anesthesia data record (ADR) include whether the patient is pacemaker-dependent, has an implantable cardioverter - defibrillator (ICD), a history of aneurysm clipping, and cochlear implants. This is especially pertinent in relation to the MRI stereotactic planning component of the surgery. Other considerations should be that anti-platelet agents should be held, if possible, and antihypertensive medications should be continued. Patients should be maintained nothing per oral (NPO) the night before the surgery, following standard preoperative fasting regimen protocols. In addition, anti-parkinsonian medications, benzodiazepines, and other GABA agonists should be withheld to optimize patient cooperation and intraoperative neuromonitoring.

\section{Summary of Anesthetic Techniques}

On the day of the surgery, placement of a head frame for accurate insertion of electrodes can be facilitated by use of either regional nerve blockade or local anesthetic infiltration at the pin sites. Watson, et al. recommend using a combined supraorbital and greater occipital nerve block as it seems to provide better results than the localized subcutaneous infiltration method [56]. In addition, added sedation or general anesthesia may not be necessary except in patients who are uncooperative or those with severe dystonia.

As microelectrode monitoring and macrostimulation serve as important conduits to target localization, gabaminergic medications have been shown to suppress the quality of the modulation error ratio (MER) signaling as evidenced by Hutchison, et al. [57]. Though no clear data exists in regards to suggestions that 'awake' technique versus general anesthesia provides better MER signaling, studies have suggested that total intravenous techniques using combination of propofol and remifentanil may in fact lead to suppression of parkinsonian tremors. In addition, other options may include using 'twilight' or monitored anesthesia care with and without sedation, analgesia, or both [58-61].

Good pain control, positioning, padding, thermal control, and excessive fluid avoidance to prevent bladder distention are all critical goals to achieve intraoperatively. Given the extreme sensitivity of the subcortical areas of the brain to GABA receptor-mediated medications, benzodiazepines should be avoided. In regards to use of propofol, studies suggest that patients with underlying Parkinson's disease may have altered pharmacokinetic profiles and may respond very differently to typical maintenance infusion rates used for sedation and general anesthesia. In addition, propofol has been shown to cause dyskinetic effects, abolish tremor, which can make surgery difficult and interfere with intraoperative testing. Use of either fentanyl and alfentanil prior to the use of propofol has also shown to reduce the occurrence of 
sneezing. This seems to be a relatively common occurrence when using propofol infusion for DBS procedures. Sneezing can cause patient discomfort, interfere with surgical mapping, and cause sudden rises in intracranial pressure putting patients at higher risks of intracranial hemorrhages, making it important to avoid intraoperatively [61-66].

Dexmedetomidine primarily influences neuronal behavior through $\alpha$-adrenoreceptors in the locus coeruleus. It has been frequently used at large centers conducting DBS placement in both adult and pediatric populations. Advantages include its ability to minimize respiratory depression while still affecting various critical brain functions, including arousal, sleep, and anxiety. Several case studies have shown very successful results with use of dexmedetomidine alone or in conjunction with propofol [67-71]. Other benefits of dexmedetomidine include decreased cerebral blood flow through direct $\alpha$-mediated smooth muscle constriction and indirectly through intrinsic neural pathways. In addition, decreased ICP can be achieved through preferential vasoconstriction of the venous side over the arterial side by the use of dexmedetomidine, making it a very suitable candidate for DBS implantation.

At present, there are no conclusive studies that suggest postoperative outcomes are better when comparing the 'awake' technique, primarily facilitated through the use of dexmedetomidine versus using general anesthesia with either inhalational of intravenous techniques. This may warrant further analysis to determine potential risk/benefits to either method in facilitating the best postoperative outcomes.

Strategies for Reducing Anesthetic-related Complications

Common intraoperative complications can include cough, sneezing, aspiration, pulmonary edema, combative behavior, bronchospasm, angina, intracranial hemorrhage, seizure, hypertension, and airway obstruction. We have listed some common strategies to deal with these potential complications.

In regards to hypertension, use of dexmedetomidine, alpha, beta, and calcium channel antagonists seem to provide suitable intraoperative blood pressure control. Prevention of venous air embolisms should be approached through Trendelenburg position, irrigation of surgical field, application of bone wax, sealing the headpins, and cauterizing any open vessels. Placement of a central line, IV fluids, and use of pressors may be needed for adequate maintenance of hemodynamic parameters [72].

For seizure prophylaxis, anticonvulsants should be available. Use of invasive blood pressure monitoring might find benefits in regards to intraoperative blood pressure control. In addition, surgical homeostasis and avoidance of coughing and sneezing may help to reduce the incidence of intracranial hemorrhages. Should airway difficulties present intraoperatively, standard Amerian Society of Anesthesiologists (ASA) guidelines for difficult airway should be resorted to, with emphasis on prompt use of repositioning and use of laryngeal mask airway.

Though we have suggested a few strategies with regards to common complications that might present intraoperatively, our recommendations are consistent with many large centers that suggest that a thorough preoperative evaluation can be the best mechanism of reducing intraand postoperative complications.

\section{Programming and engineering intracranial electrodes and pulse generators}

The beauty of DBS is its inherent synthesis of man and machine. Beyond specific neuroanatomical substrate stimulation, surgical technique, and imaging, the basic mechanical and 
biomedical engineering of the DBS system is of vital importance to the future of functional neurosurgery and DBS in general. Intracranial electrode leads connected to extension wires funneled subcutaneously to an implanted pulse generator (IPG) underneath the collar bone comprise the basic 'DBS System'. The functionality of the IPG is imperative to the overall operating system, as it sends a specific electric signal to the brain at a particular pulse width, frequency, and amplitude set by the neurosurgeon. Initially accepted DBS systems included voltage-controlled IPGs which meant that electric current delivered to the brain was inherently dependent on electrode impedance. This presented an inherent limitation in stimulation as electrochemical alterations naturally occurring at the lead-brain interface would yield electric current variations [73]. Consequently, the clinical implications of this particular DBS system on a neuronal level would vary because the current delivered would determine the volume and region of brain tissue being stimulated [73].

In order to circumvent this shortcoming, biotechnology companies are beginning to design and implement new IPG systems which are current-controlled. Okun, et al. completed a clinical trial in 2012 demonstrating comparable clinical efficacy in patients when using newly developed current-controlled IPG systems in DBS instead of voltage-controlled IPGs [74]. In order to provide a patient with the maximum efficacy via DBS treatment, it is important that the programming of the DBS system be set appropriately. Programming the DBS system entails selecting electrical stimulation parameters and boundaries which will provide the most effective electrical outcome, while simultaneously sparing the stimulation of unnecessary neuronal structures and circuits. The science of neurosurgical practice has been limited in its ability to go beyond the trial and error phases of obtaining the optimal electrode contact, amplitude of stimulation, pulse width, and frequency. Conventional neurosurgical practice entails an exhaustive process of testing each electrical contact zone in various combinations of stimulation guidelines without an objective baseline of reference to assess the appropriate target. Essentially, under these given circumstances, the neurosurgical team is charged with the responsibility to subjectively assess the clinical benefit of each stimulation scenario and the risk-benefit analysis associated with the conjunctive adverse effects.

To circumvent this issue, new computational modalities have been invented and introduced into the surgical planning for DBS which have the capacity to determine the optimal stimulation parameters in concordance with electrode location and the aimed stimulation target (Figure 1). These computer techniques also determine optimal parameters of stimulation based on determining and assessing the spread of the potentially administered current throughout adjacent brain tissue. Postoperative magnetic resonance imaging (MRI) is used to determine the precise location of the implanted electrodes in a safe and effective manner. An alternate method proposed by Chhabra, et al. postulates the inculcation of preoperative MRI with postoperative computer tomography [75]. Regions of stimulation within the brain may also be dictated by prior clinical experience which incorporates the limitations and advantages of DBS in a particular region for specific symptom alleviation. With evolving data correlating neuronal substrates with DBS appropriate targets, computational models may be further developed to calculate the precise current to be induced via the implanted electrodes, while predicting its potential influence on neighboring neurons. A prediction-based computer model for DBS treatment of a host of diseases is valuable in that it may be utilized to obtain parameters and guidelines for electrical input in a given clinical scenario, which can protect adjacent healthy brain tissue while providing the maximum threshold of electric input to the targeted computed area of interest.

A safety issue to be taken into surgical consideration in DBS treatment is that the electric field emanating from the electrodes spreads in an indiscriminate manner throughout the brain region in contact with the electrode. Consequently, unwarranted neuronal stimulation may result in geographic regions not involved in the neuropathophysiology of the disease being treated. A rising innovation to circumvent this issue is the introduction of ontogenetic 


\section{Cureus}

techniques in the field of DBS. Optogenetics offers higher specificity than conventional DBS via electricity. By utilizing light, these techniques enable the stimulation of specific neurons at a higher level of specificity than conventional electric DBS. As Gradinaru, et al. report, this method can be used in the brain by introducing a viral vector aimed to select particular neuronal regions and introduce genes, which enable light-sensitive excitation of the ion channels on the targeted neurons [76]. Although futuristic in nature, animal models have been successfully used with optogenetic DBS techniques.
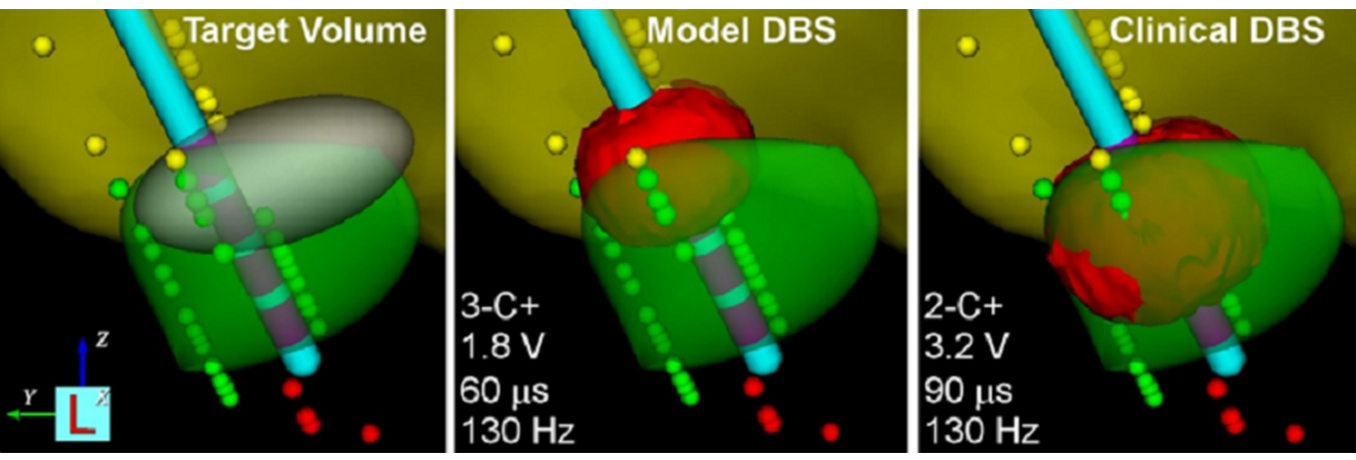

\section{FIGURE 1: Computational Electrode Placement}

Adapted with permission from Cameron McIntyre 2013.

\section{Imaging of the brain in deep brain stimulation}

\section{Positron Emission Tomography}

Resting state H215O PET of the globus pallidus interna (GPi) in Parkinson's disease patients with DBS have shown conflicting results with early work by Davis, et al. demonstrating increased regional cerebral blood flow (rCBF) in the ipsilateral supplementary motor area (SMA), globus pallidus externa (GPe), putamen, and parahippocampal gyrus while the contralateral anterior cingulate cortex showed decreasing rCBF [77]. However, later studies concluded that GPi DBS had either no effect on rCBF or that $\mathrm{rCBF}$ was decreased in the putamen, SMA, premotor cortex, and sensorimotor cortex (SMC) [78-79]. Work using 2-deoxy2-(18F) fluoro-D-glucose (FDG) PET and multivariate analysis found that GPi DBS resulted in decreased Parkinson's disease-related motor pattern (PDRP) expression ipsilateral to the site of stimulation [80]. This was correlated with improved motor symptoms and showed that normalized activity of the PDRP network through the classical cortico-striato-pallidothalamocortical motor loop underlies the benefit of GPi stimulation.

Increased rCBF and FDG uptake has been found in the stimulation site in subthalamic nucleus (STN) DBS [81-83]. As in the case of GPi DBS, subthalamic nucleus (STN) DBS also results in decreased PDRP net activity and improved motor symptoms [84-85].

PET studies of thalamic DBS involving the ventral intermediate nucleus (VIM) for treatment of Parkinson's disease tremor have shown decreased rCBF in the ipsilateral SMA, caudate, sensorimotor cortex, and bilateral cerebellum, while increased activity was later reported in frontal regions [86-88]. A recent study of VIM DBS using network analysis determined a Parkinson's disease tremor-related metabolic pattern (PDTP) composed of increased metabolism in the cerebellum/dentate nucleus, primary motor cortex, and putamen/caudate [89].

Activation PET studies have suggested that normalization of hyperactivity in motor-related 
cortical regions underlies the effect on motor (GPi or STN DBS) and speech (STN DBS) [90]. This supports the theory that excessive inhibitory output from the GPi resulting from hyperactive spontaneous firing of the STN results in impairment of desired motor pathways while at the same time allowing activation of unselected motor networks.

\section{Magnetic Resonance Imaging}

Safety consideration of MRI after DBS placement is important, given the complex interaction between implanted DBS hardware and MRI scanner electromagnetic fields [91]. Serious injury and even death may result from heating, movement, and electrical current in the implanted cables and electrodes. Nevertheless, after careful consideration of safety issues, postoperative MRI after DBS placement has been safely used by many centers to determine accurate lead placement and complications, such as intracranial hemorrhage [92].

\section{Functional MRI (fMRI)}

An early report by Rezai, et al. in 1999 demonstrated that fMRI could safely be performed in patients with implanted DBS electrodes [93]. The pulse sequences for fMRI produce magnetic fields of larger magnitude that could cause harm to pulse generators and active DBS contacts. fMRI studies can be performed with low risk by using externalized leads and having the pulse generators removed from the MR field [94]. Little is known regarding blood oxygen leveldependent (BOLD) signal changes with DBS. In 2003, Stefurak, et al. published a case report which appeared to demonstrate lateralized BOLD signal changes with DBS in mood and motor circuits [94]. While these methods may provide insight into functional connectivity between cortical loops and the basal ganglia, the interpretation of these findings relies heavily on the accurate knowledge of the location of DBS site and effects [95]. In 2004, Hesselmann, et al. demonstrated the feasibility of intraoperative fMRI to monitor the effect of DBS in a patient with Parkinson's disease. Further validation is necessary; however, the results of this study raise the possibility that intraoperative fMRI could serve as a means to optimize DBS electrode placement [96].

\section{Diffusion Tensor Imaging (DTI)}

DTI is a form of functional MRI technique that allows one to describe water diffusion directionality to noninivasively determine structural integrity of neural tissue and visualize the organization of white matter fiber tracts [97]. DTI can provide insight into the mechanisms of DBS by characterizing its effects on neighboring tissues.

The primary white matter fiber bundle that comprises the superior cerebellar peduncle is the dentatorubrothalamic tract (DRT) [98]. Based on superficial (thalamic) and inferior (posterior subthalamic) DBS target regions, tremor reduction has been theorized to be related to involvement of the DRT and recent work with DTI fiber tracking (FT) appears to substantiate this, though further validation remains necessary to verify this hypothesis [99-100].

Hypomania is reported to occur in $4 \%-13 \%$ of cases during STN DBS for treatment of Parkinson's disease [101]. This has often been attributed to the diffusion of current into the inferior and medial STN, although recent work with DTI has suggested that the medial forebrain bundle (MFB) may be responsible, and as such, it may serve as a potential target for DBS in the treatment of refractory major depression [102]. The MFB is an important structure of reward-seeking circuitry. The targeting of the nucleus accumbens, anterior limb of the internal capsule, and subgenual cingulum with DBS has recently been used in the treatment of major depression, and there is recent DTI evidence to show that these regions are interconnected by the MFB [102]. 


\section{Conclusions}

Surgical intervention within the most complex organ in the human body remains an exciting and ever-changing domain of medicine. Progress made in the last decade demonstrates the feasibility of highly intricate neurosurgical procedures aimed at curing a variety of debilitating disease states. The introduction of newly engineered surgical devices, as well as improvements in brain imaging modalities, will pave the way for a future of neurosurgery which includes deep brain stimulation interventions for a host of neuropathophysiologic-based disorders. A greater understanding of the causative relationship existing between neuronal substrates and clinical manifestation of motor, behavior, and mental disorders are necessary to propel this field of clinical medicine forward. Understanding the molecular, biochemical, and electrical properties of the brain as they relate to a variety of diseases is of utmost importance for the field of neuroscience, and the biomedical community as a whole. This goal requires an interdisciplinary and collaborative approach from bioengineers, computer scientists, physicists, and clinicians from a multitude of specialties. Hence, in the future, we will certainly see the barriers separating these domains of medicine and science dissipate in order to accomplish the very noble goal of understanding the human brain.

\section{Additional Information}

\section{Disclosures}

Conflicts of interest: In compliance with the ICMJE uniform disclosure form, all authors declare the following: Payment/services info: All authors have declared that no financial support was received from any organization for the submitted work. Financial relationships: All authors have declared that they have no financial relationships at present or within the previous three years with any organizations that might have an interest in the submitted work. Other relationships: All authors have declared that there are no other relationships or activities that could appear to have influenced the submitted work.

\section{References}

1. Hardenacke K., Shubina E, Burle CP, et al.: Deep brain stimulation as a tool for improving cognitive function in Alzheimer’z dementia: A systematic review. Front Psychiatry. 2013, $4: 159$.

2. Kern D, Kumar R: Deep brain stimulation. Neurologist. 2007, 13:237-52.

3. Hassler R, Riechart T, Munginer F, Umbach W, Ganglberger JA: Physiological observations in stereotaxic operations in extrapyramidal motor disturbances. Brain. 1960, 83:337-50.

4. Spiegel EA, Wycis HT: Thalamotomy and pallidotomy for treatment of choreic movements . Acta. Neurochir. (Wien). 1952, 2:417- 22.

5. Hosobuchin Y, Adams JE, Rutkin B: Chronic thalamic stimulation for the control of facial anesthesia dolorosa. Arch. Neurol. 1973, 29:158-61.

6. Cooper IS: Effect of chronic stimulation of anterior cerebellum on neurological disease . Lancet. 1973, 1:206.

7. Brice J, McLellan L: Suppression of intention tremor by contingent deep-brain stimulation . Lancet. 1980, 1:1221-22.

8. Perlmutter SJ, Mink JW: Deep brain stimulation. Annu Rev Neurosci. 2006, 29:229-57.

9. Carelli RM: Nucleus accumbens cell firing and rapid dopamine signaling during goal-directed behaviors in rats. Neuropharmacology. 2004, 47:180-9.

10. Stocco A, Leviere C, Anderson JR: Conditional routing of information to the cortex: a model of the basal ganglia's role in cognitive coordination. Psychol Rev. 2010, 117:541-74. 10.1037/a0019077

11. Kreitzer AC, Malenka RC: Striatal plasticity and basal ganglia circuit function. Neuron. 2008, 60:543-54. 10.1016/j.neuron.2008.11.005

12. Guridi J, Rodriguez-Oroz MC, Lozano AM, Moro E, Albanese A, Nuttin B, Gybels J, Ramos E, Obeso JA: Targeting the basal ganglia for deep brain stimulation in Parkinson's disease . 
Neurology. 2000, 55:S21-8.

13. Miocinovic S, Somayajula S, Chitnis S, Vitek JL: History, applications, and mechanisms of deep brain stimulation. JAMA Neurol. 2013, 70:163-71. 10.1001/2013.jamaneurol.45

14. Bell E, Mathieu G, Racine E: Preparing the ethical future of deep brain stimulation . Surg Neurol. 2009, 72:577-86.

15. Bourne SK, Eckhardt CA, Sheth SA, Eskandar EN: Mechanisms of deep brain stimulation for obsessive compulsive disorder: Effects upon cells and circuits. Front Integr Neurosci. 2012, 6:29. 10.3389/fnint.2012.00029

16. Viswanathan A, Jimenez-Shahed J, Carvallo JF, Jankovic J: Deep brain stimulation for Tourette Syndrome: Target selection. Stereotact Funct Neurosurg. 2012, 90:213-24. 10.1159/000337776

17. Taghva AS, Malone DA, Rezai AR: Deep brain stimulation for treatment-resistant depression . World Neurosurg. 2013, 80:S27.e17-24. 10.1016/j.wneu.2012.10.068

18. Kumar R, Lozano AM, Montgomery E, Lang AE: Pallidotomy and deep brain stimulation of the pallidum and subthalamic nucleus in advanced Parkinson's disease. Mov Disord. 1998, 13:7382 .

19. Bergman H, Deuschl G: Pathophysiology of Parkinson's disease: From clinical neurology to basic neuroscience and back. Mov Disord. 2002, 17:S28-40.

20. Oroz MC, Obeso JA, Lang AE, et al.: Bilateral deep brain stimulation in Parkinson's disease: A multicentre study with 4 years follow-up. Brain. 2005, 128:2240-9.

21. Kumar R, Lozano AM, Sime E, Lang AE: Long-term follow-up of thalamic deep brain stimulation for essential and parkinsonian tremor. Neurology. 2003, 61:1601-04.

22. Anderson VC, Burchiel KJ, Hogarth P, Favre J, Hammerstad JP: Pallidal vs subthalamic nucleus deep brain stimulation in Parkinson disease. Arch Neurol. 2005, 62:554-60.

23. Loher TJ, Burgunder JM, Weber S, Sommerhalder R, Krauss JK: Effect of chronic pallidal deep brain stimulation on off period dystonia and sensory symptoms in advanced Parkinson's disease. J Neurol Neurosurg Psychiatr. 2002, 73:395-99.

24. Benabid AL, Benazzouz A, Hoffmann D, Limousin P, Krack P, Pollak P: Long-term electrical inhibition of deep brain targets in movement disorders. Mov. Disord. 1998, 13:119-25.

25. Bastian AJ, Kelly VE, Revilla FJ, Perlmutter JS, Mink JW: Different effects of unilateral versus bilateral subthalamic nucleus stimulation on walking and reaching in Parkinson's disease. Mov Disord. 2003, 18:1000-7.

26. Rizzone M, Ferrarin M, Pedotti A, Bergamasco B, Bosticco E, et al.: High-frequency electrical stimulation of the subthalamic nucleus in Parkinson's disease: kinetic and kinematic gait analysis. Neurol Sci. 2002, 23:S103-4.

27. Ferrarin M, Rizzone M, Bergamasco B, Lanotte M, Recalcati M, et al.: Effects of bilateral subthalamic stimulation on gait kinematics and kinetics in Parkinson's disease. Exp. Brain. 2005, 160:517- 27.

28. Kumar R, Dagher A, Hutchinson W, Lang AE, Lozano AM: Globus pallidus deep brain stimulation for generalized dystonia: Clinical and PET investigation. Neurology. 1999, 53:8714.

29. Vitek JL: Long-term benefit from deep brain stimulation of the subthalamic nucleus: Is it for everyone?. Alzheimers Res Ther. 2012, 4:13. 10.1186/alzrt111

30. Hershey T, Revilla F, Wernle A, Schneider Gibson P, Dowling J, Perlmutter JS: Stimulation of STN impairs aspects of cognitive control in PD. Neurology. 2004, 62:1110-14.

31. Jahanshahi M, Ardouin CM, Brown RG, et al.: The impact of deep brain stimulation on executive function in Parkinson's disease. Brain. 2000, 123:1142-54.

32. Herzog J, Reiff J, Krack P, Witt K, Schrader B,et al.: Manic episode with psychotic symptoms induced by subthalamic nucleus stimulation in a patient with Parkinson's disease. Mov Disord. 2003, 18:1382-84.

33. Diederich NJ, Alesch F, Goetz C: Visual hallucinations induced by deep brain stimulation in Parkinson's disease. Clin Neuropharmacol. 2000, 23:287-9.

34. Berney A, Vingerhoets F, Perrin A, Guex P, Villemure JG, et al.: Effect on mood of subthalamic DBS for Parkinson's disease: a consecutive series of 24 patients. Neurology. 2002, 59:1427-29.

35. Sheth SA, Mian MK, Abuelem T, Gale JT, Eskandar EN: Facilitation of visuomotor associative learning by the basal ganglia. Clin Neurosurg. 2010, 57:145-50.

36. Berardelli A, Rothwell JC, Hallett M, Thompson PD, Manfredi M, Marsden CD: The pathophysiology of primary dystonia. Brain. 1998, 121:1195-212.

37. Mentzel CL, Tenback DE, Tijssen MA, Visser-Vandewalle VE, van Harten PN: Efficacy and 
safety of deep brain stimulation in patients with medication-induced tardive dyskinesia and/or dystonia: A systematic review. J Clin Psychiatry. 2012, 73:1434-8.

10.4088/JCP.12r07643

38. Vitek JL, Zhang J, Evatt M, et al.: GPI pallidotomy for dystonia: Clinical outcome and neuronal activity. Adv Neurol. 1998, 78:211-219.

39. Ford B: Pallidotomy for generalized dystonia. Adv Neurol. 2004, 94:287-299.

40. Benabid AL, Benazzouz A, Hoffmann D, Limousin P, Krack P, Pollak P: Long-term electrical inhibition of deep brain targets in movement disorders. Mov Disord. 1998, 13:119-125.

41. Jankovic J: Re-emergence of surgery for dystonia. J Neurol Neurosurg Psychiatry. 1998, 65:434.

42. Lang AE: Surgical treatment of dystonia. Adv Neurol. 1998, 78:185-198.

43. Krauss JK: Deep brain stimulation for dystonia in adults: Overview and developments . Stereotact Funct Neurosurg. 2002, 78:168-182.

44. Lozano AM, Abosch A: Pallidal stimulation for dystonia. Adv Neurol. 2004, 94:301-08.

45. Vercueil L, Krack P, Pollak P: Results of deep brain stimulation for dystonia: A critical reappraisal. Mov Disord. 2002, 17:S89-93.

46. Vidailhet M, Vercueil L, Houeto JL, Krystkowiak P, Benabid AL, et al.: Bilateral deep brain stimulation of the globus pallidus in primary generalized dystonia. NEJM. 2005, 352:459-67.

47. Kiss ZH, Doig K, Eliasziw M, Ranawaya R, Suchowersky O: The Canadian multicenter trial of pallidal deep brain stimulation for cervical dystonia: preliminary results in three patients. Neurosurg. Focus. 2004, 17:E5.

48. Castelnau P, Ci fL, Valente EM, Vayssiere N, Hemm S, et al.: Pallidal stimulation improves pantothenatekinase-associated neurodegeneration. Ann. Neurol. 2005, 57:738-41.

49. Hasler G: Pathophysiology of depression: Do we have any solid evidence of interest to clinicians?. World Psychiatry. 2010, 9:155-61.

50. Lozano AM, Mayberg HS, Giacobbe P, Hamani C, Craddock RC, Kennedy SH: Subcallosol cingulate gyrus deep brain stimulation for treatment-resistant depression. Biological Psychiatry. 2008, 64:461-7.

51. Taghva AS, Corrigan JD, Rezai AR: Obesity and brain addiction circuitry: Implications for deep brain stimulation. Neurosurg. 2012, 71:224-38.

52. Nauczyciel C, Robic S, Dondaine T, Verin M, Robert G, Drapier D, Naudet F, Millet B: The nucleus accumbens: A target for deep brain stimulation in resistant major depressive disorder. J Molecular Psychiat. 2013, 1:1-12.

53. Voorn P, Vanderschuren LJ, Groenewegen HJ, Robbins TW, Pennartz CM: Putting a spin on the dorsal-ventral divide of the striatum. Trends Neurosci. 2004, 27:468-74.

54. Poon CC, Irwin MG: Anaesthesia for deep brain stimulation and in patients with implanted neurostimulator devices. Br J Anaesth. 2009, 103:152-65. 10.1093/bja/aep179

55. Baltuch GH, Stern MB: Surgical Management of Movement Disorders. Taylor \& Francis, Boca Raton; 2005.

56. Watson R, Leslie K: Nerve blocks versus subcutaneous infiltration for stereotactic frame placement. Anesth Analg. 2001, 92:424-7.

57. Hutchison WD, Lozano AM: Microelectrode recordings in movement disorder surgery. Movement Disorder Surgery. Lozano AM (ed): Karger, Basel; 2000. 103-17.

58. Böhmdorfer W, Schwarzinger P, Binder S, Sporn P: Temporary suppression of tremor by remifentanil in a patient with Parkinson's disease during cataract extraction under local anesthesia (Article in German). Anaesthesist. 2003, 52:795-7.

59. Burton DA, Nicholson G, Hall GM : Anaesthesia in elderly patients with neurodegenerative disorders: Special considerations.. Drugs Aging. 2004, 21:229-42.

60. Krauss JK, Akeyson EW, Giam P, Jankovic J: Propofol-induced dyskinesias in Parkinson's disease. Anesth Analg. 1996, 83:420-2.

61. Venkatraghavan L, Manninen P, Mak P, Lukitto K, Hodaie M, Lozano A: Anesthesia for functional neurosurgery: Review of complications. J Neurosurg Anesthesiol. 2006, 18:64-7.

62. Fabregas N, Rapado J, Gambus PL, et al.: Modeling of the sedative and airway obstruction effects of propofol in patients with Parkinson disease undergoing stereotactic surgery. Anesthesiology. 2002, 97:1378-86.

63. Deogaonkar A, Deogaonkar M, Lee JY, Ebrahim Z, Schubert A: Propofol-induced dyskinesias controlled with dexmedetomidine during deep brain stimulation surgery. Anesthesiology. 2006, 104:1337-9. 
64. Krauss JK, Akeyson EW, Giam P, Jankovic J: Propofol-induced dyskinesias in Parkinson's disease. Anesth Analg. 1996, 83:420-2.

65. Anderson BJ, Marks PV, Futter ME: Propofol-contrasting effects in movement disorders . Br J Neurosurg. 1994, 8:387-8.

66. Tao J, Nunery W, Kresovsky S, Lister L, Mote T: Efficacy of fentanyl or alfentanil in suppressing reflex sneezing after propofol sedation and periocular injection. Ophthal Plast Reconstr Surg. 2008, 24:465-7.

67. Almeida AN, Tavares C, Tibano A, Sasaki S, Murata KN, Marino R, Jr: Dexmedetomidine for awake craniotomy without laryngeal mask. Arq Neuropsiquiatr. 2005, 63:748-50.

68. Bekker AY, Kaufman B, Samir H, Doyle W: The use of dexmedetomidine infusion for awake craniotomy. Anesth Analg. 2001, 92:1251-3.

69. Mack PF, Perrine K, Kobylarz E, Schwartz TH, Lien CA: Dexmedetomidine and neurocognitive testing in awake craniotomy. J Neurosurg Anesthesiol. 2004, 16:20-25.

70. Ard J, Doyle W, Bekker A: Awake craniotomy with dexmedetomidine in pediatric patients. J Neurosurg Anesthesiol. 2003, 15:263-6.

71. Rozet I: Anesthesia for functional neurosurgery: The role of dexmedetomidine . Curr Opin Anaesthesiol. 2008, 21:537-43.

72. Palmon SC, Moore LE, Lundberg J, Toung T: Venous air embolism: A review . J Clin Anesth. 1997, 9:251-7.

73. Miocinovic S, Lempka SF, Russo GS, et al.: Experimental and theoretical characterization of the voltage distribution generated by deep brain stimulation. Exp Neurol. 2009, 216:166-76.

74. Okun MS, Gallo BV, Mandybur G, et al. : Subthalamic deep brain stimulation with a constantcurrent device in Parkinson's disease: an open label randomised controlled trial. Lancet Neurol. 2012, 11:140-49.

75. Chhabra V, Sung E, Mewes K, Bakay RA, Abosch A, Gross RE: Safety of magnetic resonance imaging of deep brain stimulator systems: a serial imaging and clinical retrospective study. J Neurosurg. 2010, 112:497-502. 10.3171/2009.7.JNS09572

76. Gradinaru V, Mogri M, Thompson KR, Henderson JM, Deisseroth K: Optical deconstruction of parkinsonian neural circuitry. Science. 2009, 324:354-359.

77. Davis KD, Taub E, Houle S, et al.: Globus pallidus stimulation activates the cortical motor system during alleviation of parkinsonian symptoms. Nature Med. 1997, 3:671-674.

78. Limousin P, Greene J, Pollak P, et al.: Changes in cerebral activity pattern due to subthalamic nucleus or internal pallidum stimulation in Parkinson's disease. Ann Neurol. 1997, 42:283291.

79. Payoux P, Remy P, Miloudi M, et al.: Contrasting changes in cortical activation induced by acute high frequency stimulation within the globus pallidus in Parkinson's disease. J Cereb Blood Flow Metab. 2009, 29:235-243.

80. Fukuda M, Mentis MJ, Ma Y, et al.: Networks mediating the clinical effects of pallidal brain stimulation for Parkinson's disease - a PET study of resting-state glucose metabolism. Brain. 2001, 124:1601-1609.

81. Hershey L, Revilla FJ, Loepker A, et al.: Cortical and subcortical blood flow effects of subthalamic nucleus stimulation in PD. Neurology. 2003, 61:816-821.

82. Geday J, Østergaard K, Johnsen E, et al.: STN-stimulation in Parkinson's disease restores striatal inhibition of thalamocortical projection. Hum Brain Mapp. 2009, 30:112-121.

83. Hilker R, Voges J, Weisenbach S, et al.: Subthalamic nucleus stimulation restores glucose metabolism in associative and limbic cortices and in cerebellum: evidence from a FDG-PET study in advanced Parkinson's disease. J Cereb Blood Flow Metab. 2004, 24:7-16.

84. Asanuma K, Tang C, Ma Y, et al.: Network modulation in the treatment of Parkinson's disease . Brain. 2006, 129:2667-2678.

85. Trost M, Su S, Su P, et al.: Network modulation by the subthalamic nucleus in the treatment of Parkinson's disease. Neuroimage. 2006, 31:301-307.

86. Parker F, Tzourio N, Blond S, Petit H, Mazoyer B: Evidence for a common network of brain structures involved in parkinsonian tremor and voluntary repetitive movement. Brain Res. 1992, 584:11-17.

87. Deiber MP, Pollak P, Passingham R, et al.: Thalamic stimulation and suppression of parkinsonian tremor. Evidence of a cerebellar deactivation using positron emission tomography. Brain. 1993, 116:267-279.

88. Davis KD, Taub E, Houle S, et al.: Globus pallidus stimulation activates the cortical motor 
system during alleviation of parkinsonian symptoms. Nature Med. 1997, 3:671-674.

89. Mure H, Hirano S, Tang CC, et al.: Parkinson's disease tremor-related metabolic network: characterization, progression, and treatment effects. Neuroimage. 2011, 54:1244-1253.

90. Ko JH, Tang CC, Eidelberg D, et al.: Brain stimulation and functional imaging with fMRI and PET. Handb Clin Neurol. 2013, 116:77-95. 10.1016/B978-0-444-53497-2.00008-5

91. Zrinzo L, Yoshida F, Hariz MI, et al.: Clinical safety of brain magnetic resonance imaging with implanted deep brain stimulation hardware: large case series and review of the literature. World Neurosurg. 2011, 76:164-172.

92. Larson PS, Richardson RM, Starr PA, Martin AJ: Magnetic resonance imaging of implanted deep brain stimulators: experience in a large series. Stereotactic and Funct Neurosurg. 2008, 86:92-100.

93. Rezai A.R., Lozano A.M., Crawley A.P, et al.: Thalamic stimulation and functional magnetic resonance imaging: localization of cortical and subcortical activation with implanted electrodes. Technical note. J Neurosurg. 1999, 90:583-590.

94. Stefurak T, Mikulis D, Mayberg H, Lang AE, Hevenor S, Pahapill P, Saint-Cyr J, Lozano A: Deep brain stimulation for Parkinson's disease dissociates mood and motor circuits: a functional MRI case study. Mov Disord. 2003, 18:1508-1541.

95. Perlmutter JS, Mink JW: Deep brain stimulation. Ann Rev Neurosci. 2006, 29:229-57.

96. Hesselman V, Sorger B, Gimus R, et al.: Intraoperative functional MRI as a new approach to monitor deep brain stimulation in Parkinson's disease. Eur Radiol. 2004, 14:686-690.

97. Lerner A, Mogensen MA, Kim PE, Shiroishi MS, Hwang DH, Law M: Clinical applications of diffusion tensor imaging. World Neurosurg. 2013 , S1878-8750. 10.1016/j.wneu.2013.07.083

98. Coenen VA, Mädler B, Schiffbauer H, et al.: Individual fiber anatomy of the subthalamic region revealed with diffusion tensor imaging: a concept to identify the deep brain stimulation target for tremor suppression. Neurosurgery. 2011, 68:1069-76.

99. Coenen VA, Allert N, Mädler B: A role of diffusion tensor imaging fiber tracking in deep brain stimulation surgery: DBS of the dentato-rubro-thalamic tract (drt) for the treatment of therapy-refractory tremor. Acta Neurochir (Wien). 2011, 153:1579-85. 10.1007/s00701-0111036-Z

100. Henderson JM: "Connectomic surgery": Diffusion tensor imaging (DTI) tractography as a targeting modality for surgical modulation of neural networks. Front Integr Neurosci. 2012, 6:15. 10.3389/fnint.2012.00015

101. Coenen VA, Schlaepfer TE, Allert N, Mädler B: Diffusion tensor imaging and neuromodulation: DTI as key technology for deep brain stimulation. Int Rev Neurobiol. 2012, 107:207-34. 10.1016/B978-0-12-404706-8.00011-5

102. Coenen VA, Panksepp J, Hurwitz TA, Urbach H, Mädler B: Human medial forebrain bundle (MFB) and anterior thalamic radiation (ATR): Imaging of two major subcortical pathways and the dynamic balance of opposite affects in understanding depression. J Neuropsychiatry Clin Neurosci. 2012, 24:223-36. 10.1176/appi.neuropsych.11080180 